\title{
EFECTOS DEL USO DEL SUELO EN LAS PROPIEDADES EDÁFICAS Y LA ESCORRENTÍA SUPERFICIAL EN UNA CUENCA DE LA ORINOQUIA COLOMBIANA
}

\author{
Effects of land use on soil properties and run-off in a colombian Orinoco \\ water basin
}

\author{
David Alejandro Sánchez Núñez ${ }^{1}$, Gabriel Antonio Pinilla² \& José Ernesto Mancera Pineda ${ }^{3}$
}

Sánchez N, D., Pinilla, G. \& Mancera P, J. (2015). Efectos del uso del suelo en las propiedades edáficas y la escorrentía superficial, en una cuenca de la Orinoquia colombiana. Colombia Forestal, 18(2),255-272.

Recepción: 18 de agosto de 2014

\section{RESUMEN}

Con el objetivo de evaluar los efectos del uso del suelo sobre la cuenca del caño Camoa, Meta, se cuantificó el contenido de materia orgánica edáfica (CMOE), la densidad aparente y el contenido de agua en condiciones de campo y capacidad de campo. Para ello se extrajeron núcleos de suelo de bosques y pastizales riparios con diferente productividad de hojarasca y se evaluó la relación entre el CMOE y el volumen deficitario de agua mediante regresiones. Así mismo, se identificó mediante trampas de caída la organización trófica de los artrópodos de la superficie del suelo. Debido a sus CMOE superiores al 5\%, los suelos con mayor cantidad de hojarasca (alta productividad) presentaron contenidos de agua superiores a los de bosques menos productivos y de pastizales ( $p<0.05$ en ambos casos). Las diferencias en el contenido de agua de los suelos de las distintas coberturas evaluadas sugieren que el papel de la escorrentía superficial en los bosques ha sido subestimado. El manejo de cuencas debe propender por el establecimiento de bosques productivos que generen $\mathrm{CMOE}$ superiores al $5 \%$, para
Aprobación: 6 de abril de 2015

promover la continuidad y el volumen en la oferta hídrica de los ríos al generar flujos constantes de agua subsuperficial y al regular flujos esporádicos superficiales. La variedad de funciones e interacciones tróficas que cumplen los artrópodos del suelo en los bosques indica que una estructuración óptima de esta comunidad favorecería la conversión de compuestos simples y complejos en humus y por lo tanto la capacidad de retención de agua.

Palabras clave: caño Camoa, humedad del suelo, Llanos Orientales, manejo de cuencas, materia orgánica, regulación hídrica

\section{ABSTRACT}

This study evaluates the effects of land use on the Camoa water basin in Meta by quantifying the effect of soil organic matter content (SOMC), bulk density and water content. These variables were evaluated by extracting ground cores from riparian forests and grasslands of different litter cover. We also evaluated the relationship between the SOMC and the water volume deficit using regressions. Likewise,

1 Departamento de Biología, Universidad Nacional de Colombia, Bogotá, Colombia. dasanchezn@bt.unal.edu.co. Autor de correspondencia

2 Departamento de Biología, Universidad Nacional de Colombia, Bogotá, Colombia. gapinillaa@unal.edu.co

Departamento de Biología, Universidad Nacional de Colombia, Bogotá Colombia. jemancerap@unal.edu.co 
using pitfall traps we identified the trophic organization of soil-superficial arthropods. Due to its higher SOMC (> 5\%), the soils of highly productive forest (high litter content) had significantly higher water contents than soils from less productive forests and grasslands ( $p<0.05$ in both cases). The water content found among the studied soil cover types suggests that the role of run-off in forest has been underestimated. Water basin management should encourage the establishment of productive riparian forests with SOMC values higher than 5\% in order to promote volume and continuity in water offer from rivers due to the generation of constant subsurface flow and the regulation of sporadic superficial run-offs. The variety of functions and interactions accomplished by arthropods in forest soils suggests that a highly structured trophic organization favors conversion of simple and complex compounds in humus and therefore would favors soil water retention capacity.

Keywords: Camoa River, soil humidity, Llanos Orientales, water basin management, organic matter, water flow regulation.

\section{INTRODUCCIÓN}

El estudio de la relación entre el cambio de uso del suelo, la calidad y la cantidad del agua ha sido un tema de mucho interés en varias regiones del mundo debido a la extensa conversión de bosques y otras coberturas naturales en pastizales y tierras de cultivo (Krishnaswamy et al., 2001; Scanlon et al., 2005; Huang et al., 2013). Además, el tipo de cobertura vegetal asociada al uso del suelo influye en la textura del mismo y determina la estructura edáfica, lo que a su vez modifica las propiedades hídricas del suelo (Kavian et al., 2014). Se ha identificado por ejemplo que a nivel de cuenca las transformaciones en el uso del suelo generan pérdidas de materia orgánica, incremento en la densidad del suelo y de la erosión, disminución en la continuidad de los flujos hídricos e incremento de la escorrentía superficial (Wei et al., 2007; MartínezMena et al., 2008; Dominati et al., 2010). Debido a la magnitud de dichas tendencias, se considera que el impacto de las transformaciones del uso del suelo sobre el ciclo del agua podrían sobrepasar aquel generado por efectos recientes del cambio climático (Vörösmarty et al., 2004).

La cobertura arbórea, particularmente, juega un papel crucial en el mantenimiento de la continuidad hídrica de muchos ríos y riachuelos al retardar la escorrentía superficial y aumentar la infiltración por el incremento de materia orgánica y raíces (Blinn \& Kilgore, 2001; Critchley \& Bruijnzeel, 1996). Sin embargo, varios estudios empíricos han mostrado que los suelos de bosques generan también altos valores de escorrentía (Wei et al., 2007). Esto muestra que el comportamiento hidrológico es más variable de lo esperado inicialmente en lo suelos boscosos, lo que además puede obedecer al contenido de humedad del suelo asociado a esta cobertura (Kavian et al., 2014).

En suma, la producción, degradación y acumulación de materia orgánica en los bosques riparios desempeña un papel relevante en la generación de una mayor continuidad y volumen de agua al modificar las propiedades hídricas del suelo. Por su parte, la dinámica de la descomposición de material vegetal y el reciclaje de la materia orgánica edáfica son procesos facilitados por varios grupos de organismos, entre los cuales se destacan los artrópodos (Lavelle, 1997). Sin embargo, el ordenamiento de cuencas y la valoración de sus servicios ecosistémicos (SE) consideran escasamente las relaciones funcionales entre los usos del suelo, el desarrollo de los ecosistemas, la materia orgánica, los organismos detritívoros y descomponedores del suelo y el SE de regulación hídrica (Rodríguez, 2006; Le Maitre et al., 2014). Esto sugiere que se requiere tener un mayor conocimiento de los procesos funcionales asociados a la regulación 
hídrica, tanto desde la perspectiva técnico-científica como desde la gestión de las agencias responsables del ordenamiento territorial. Así mismo, se debe conocer cómo operan dichos procesos funcionales en diferentes usos del suelo.

En muchos municipios de la Orinoquia colombiana, como es el caso de San Martín de los Llanos, localizado en la cuenca del caño Camoa, el manejo de las hoyas hidrográficas ha estado determinado por el crecimiento de las actividades agropecuarias, especialmente la ganadería (Guerrero \& Arias, 1984; Alcaldía de San Martín de los Llanos, 1996). Para el año de 1991, parte de la cuenca estaba deforestada, por lo que empezó a cobrar valor la recuperación del caño y la reforestación de sus rondas. Además, a partir de dicha fecha el caño dejó de ser la fuente de abastecimiento de agua del municipio debido al deterioro de la calidad, volumen y continuidad de la oferta hídrica. La pérdida del caudal del caño (Cormacarena, 2004) seguramente estimuló la reforestación de las rondas, lo que ha generado la recuperación parcial de la oferta hídrica de la cuenca. De acuerdo con sus habitantes, la corriente principal no se seca aún en los periodos de baja precipitación.

Los cambios en el manejo de la cuenca del caño Camoa han condicionado la presencia de diferentes coberturas riparias con pastizales, bosques de diferente desarrollo sucesional y cultivos. De esta manera, esta cuenca constituye un buen modelo para identificar las relaciones funcionales entre la materia orgánica, las propiedades hídricas del suelo y la fauna de artrópodos, asociadas a los diferentes usos del suelo. Su estudio podría contribuir a la implementación de planes eficientes de manejo de las cuencas y de valoración de SE. Por lo tanto, los objetivos específicos de este estudio fueron: (1) evaluar la relación entre el uso del suelo ripario, el contenido en peso de materia orgánica edáfica (CMOE), la humedad del suelo y la capacidad de campo; y (2) identificar la organización trófica de la fauna de artrópodos asociada a la descomposición de la materia orgánica en suelos superficiales de bosques y pastizales en la zona riparia del caño.

\section{MATERIALES Y MÉTODOS}

\section{Área de estudio}

El caño Camoa nace en una terraza alta en el noroccidente del municipio de San Martín de los Llanos, hacia los 375 m de altitud, y desde allí lo recorre de norte a sur (IGAC, 1996). En la cuenca media cerca del casco urbano, el caño cambia de rumbo para drenar hacia el oriente, y desde allí prosigue hasta desembocar en el caño Chunaipo, en la región central del municipio, hacia los 250 $\mathrm{m}$ de altitud. Las subcuencas de estos caños hacen parte de la cuenca del río Metica y de la macrocuenca del río Meta, que tiene según el $I A v H$ (2006) un tamaño de 107025.7 km². El municipio, al igual que la región de los Ilanos colombianos, presenta un régimen de lluvias unimodal con un periodo lluvioso que va de mayo a octubre y uno seco de noviembre a abril. La temperatura media es de $25.6{ }^{\circ} \mathrm{C}$ con una variación de $\pm 2.2{ }^{\circ} \mathrm{C}$ entre los meses del año (Figura 1). El estudio se llevó a cabo al final del periodo lluvioso entre el 3 y el 6 de noviembre del 2006.

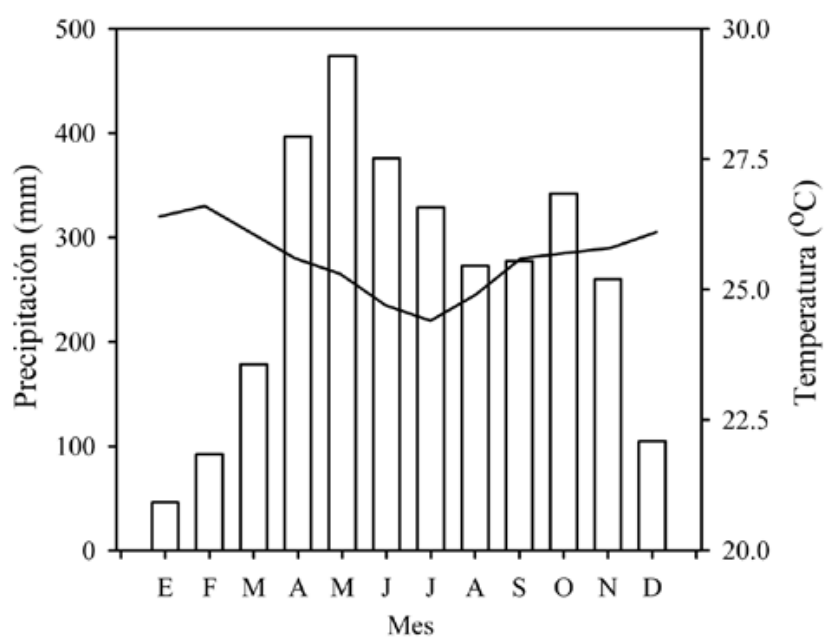

Figura 1. Precipitación (barras) y temperatura mensual promedio (línea) entre 1992 y 2006 registrada en San Martín de los Llanos según la estación climática de Barbascal del IDEAM $\left(3^{\circ} 42^{\prime} \mathrm{N}-73^{\circ} 24^{\prime} \mathrm{W}\right.$; código 3501505). 
Los muestreos se realizaron entre las 9 am y las 2 pm en cuatro sitios de la cuenca alta y media del caño Camoa (Figura 2). Las estaciones E1, E2 y E3 correspondieron a sitios de bosques secundarios siempreverdes, dado el régimen de precipitación de la zona y el alto grado de saturación de los suelos riparios (Rodríguez et al., 2010). La estación E4 se ubicó en una zona de pastizal abierto (Tabla 1).



Figura 2. Localización de las estaciones de muestreo. E1, E2 y E3 corresponden a sitios de bosques. E4 se ubicó en un pastizal abierto. 


\section{Contenido de materia orgánica, densidad aparente y variables hídricas del suelo}

Se extrajeron núcleos del suelo a unos pocos metros del cauce con un nucleador de PVC de $9 \mathrm{~cm}$ de diámetro. Estos núcleos se impermeabilizaron con papel vinilo flexible y se aislaron con bolsas plásticas resellables. En total se extrajeron tres núcleos en cada una de las estaciones, a profundidades entre 15 y $25 \mathrm{~cm}$ a partir de la superficie. En la E2 no se extrajeron núcleos por corresponder a suelos muy afectados por la actividad urbana.

De cada núcleo se tomaron tres muestras de suelo de volumen estándar $\left(113.1 \mathrm{~cm}^{3}\right)$, una en la superficie $(0-5 \mathrm{~cm})$, otra en la zona media (5-10 $\mathrm{cm}$ ) y otra en la parte profunda del núcleo (10$15 \mathrm{~cm}$ ). Estas muestras se pesaron en húmedo y hasta alcanzar peso constante luego de saturación, para estimar el contenido volumétrico de agua en el suelo, tanto en condiciones de campo como de capacidad de campo (Bashour \& Sayegh, 2007). La densidad aparente del suelo se estimó de acuerdo con Elliot et al. (1999). El CMOE se estimó para las tres profundidades mencionadas por medio del método de pérdida por ignición (Schulte \& Hopkins, 1996). Así mismo, en cada estación de muestreo se estimó el porcentaje de cubrimiento de la hojarasca sobre el suelo mediante valoración visual directa de manera similar a Kluber et al. (2008), como aproximación a la productividad del componente arbóreo de las estaciones.

\section{Identificación de la estructura trófica de los artrópodos del suelo superficial}

En cada una de las estaciones de muestreo se colocaron durante 24 horas 10 trampas de caída para insectos, en un transecto de 10 metros paralelo al cauce del caño. Por medio del conteo y medición de los individuos por especie, se estimó el peso seco de los grupos tróficos de acuerdo con la relación entre longitud del cuerpo y peso seco propuesta por Rogers et al. (1976); los resultados se expresaron en porcentaje. Los arácnidos se determinaron hasta orden, los Chalcidoideos (Hymenoptera), hasta superfamilia y las hormigas, hasta género con la colaboración de especialistas. El resto de los artrópodos se determinó a nivel de familia y morfoespecie. Cada morfoespecie se asignó a la categoría trófica más probable de acuerdo con Laurence (1955), Cloarec \& Rivault (1991), Castaño-Meneses et al. (2004), Donovan et al. (2004) y Triplehorn y Jhonson (2005); los grupos tróficos de los géneros de hormigas se asignaron de acuerdo con Fernández (2003) (Anexo 1). Las muestras de las estaciones boscosas se agruparon para tener una mayor representatividad de la fauna de artrópodos por tipo de cobertura vegetal.

Tabla 1. Localización y cobertura de las estaciones de muestreo

\begin{tabular}{|c|c|c|c|}
\hline Estación de muestreo & Ubicación & Localización geográfica & Cobertura dominante \\
\hline E1 & $\begin{array}{l}\text { Finca Las Dalias, en la vereda } \\
\text { Nora, cerca del nacimiento } \\
\text { del caño }\end{array}$ & $\begin{array}{l}3^{\circ} 44^{\prime} 10.1^{\prime \prime} \mathrm{N}- \\
73^{\circ} 42^{\prime} 53.0^{\prime \prime} \mathrm{W}\end{array}$ & $\begin{array}{l}\text { Bosque secundario siempre- } \\
\text { verde }\end{array}$ \\
\hline E2 & $\begin{array}{l}\text { Casco urbano del municipio } \\
\text { de San Martín }\end{array}$ & $\begin{array}{l}3^{\circ} 41^{\prime} 21.4^{\prime \prime} \mathrm{N}- \\
73^{\circ} 41^{\prime} 53.0^{\prime \prime} \mathrm{W}\end{array}$ & $\begin{array}{l}\text { Bosque secundario siempre- } \\
\text { verde }\end{array}$ \\
\hline E3 & $\begin{array}{l}\text { Lugar de construcción de la } \\
\text { planta de tratamiento de aguas } \\
\text { residuales del municipio }\end{array}$ & $\begin{array}{l}3^{\circ} 40^{\prime} 57.4^{\prime \prime} \mathrm{N}- \\
73^{\circ} 41^{\prime} 11.2^{\prime \prime} \mathrm{W}\end{array}$ & $\begin{array}{l}\text { Bosque secundario siempre- } \\
\text { verde }\end{array}$ \\
\hline E4 & Reserva El Caduceo & $\begin{array}{l}3^{\circ} 40^{\prime} 16.8^{\prime \prime} \mathrm{N}- \\
73^{\circ} 39^{\prime} 45.4^{\prime \prime} \mathrm{W}\end{array}$ & Pastizal \\
\hline
\end{tabular}




\section{Análisis de los datos}

El CMOE se estimó en kg. $\mathrm{m}^{-2}$ entre $0-20 \mathrm{~cm}$ de profundidad para permitir comparaciones con otros estudios. Como el CMOE en pastos y pastizales cambia con la profundidad de manera no lineal (Jobbagy \& Jackson, 2000; Porto et al., 2003), se utilizaron regresiones logarítmicas derivadas de los datos de las profundidades medidas para estimar el CMOE entre $15-20 \mathrm{~cm}$ de profundidad.

Por medio de pruebas ANOVA de dos factores se identificaron diferencias significativas en la densidad aparente del suelo y el contenido volumétrico de agua entre profundidades y estaciones de muestreo, tanto para condiciones de campo como de capacidad de campo en laboratorio, previa comprobación de normalidad y homocedasticidad en los datos. También se identificaron diferencias entre estaciones en las variables mencionadas con la prueba post hoc de diferencia significativa mínima de Fisher (LSD). Para evaluar diferencias significativas entre estaciones y profundidades en el porcentaje de materia orgánica edáfica se utilizó la prueba no paramétrica de dos factores de Scheirer Ray Hare (SRH) (Sokal \& Rolf, 1995), dado el incumplimiento del supuesto de homogeneidad de la varianza. En este último caso, se identificaron diferencias significativas entre grupos de un mismo factor por medio de la prueba $U$ de Mann Whitney, seguida de la corrección de Bonferroni (Moran, 2003). Para el análisis estadístico se utilizó el programa Statgraphics (Statpoint Technologies Inc., 1998).

Para evaluar la relación entre el CMOE y el contenido de agua en el suelo presente en condiciones de campo, se realizaron regresiones simples considerando como función del porcentaje de materia orgánica edáfica: (1) el volumen deficitario de agua (VDA) y (2) el porcentaje de volumen deficitario de agua (\%VDA); $\mathrm{VDA}=\mathrm{V}_{\mathrm{CC}}-\mathrm{V}_{\mathrm{C}^{\prime}} \% \mathrm{VDA}=$ $\left(\left(\mathrm{V}_{\mathrm{CC}}-\mathrm{V}_{\mathrm{C}}\right) / \mathrm{V}_{\mathrm{CC}}\right) * 100$, donde $\mathrm{V}_{\mathrm{CC}}$ es el volumen de agua en capacidad de campo y $\mathrm{V}_{\mathrm{C}}$ es el volumen en condiciones de campo.

\section{RESULTADOS}

\section{Contenido de materia orgánica, densidad aparente y variables hídricas del suelo}

La cobertura de hojarasca en el suelo ripario de E1 $(80 \%)$ fue mayor respecto a E2 (40\%) y E3 $(10 \%)$. En E4 había árboles dispersos y la cobertura de hojarasca sobre el pastizal era mínima (1\%). El análisis de SRH indica que el porcentaje de materia orgánica edáfica difirió significativamente entre estaciones $(p<0.001)$. Así mismo, el análisis de varianza señaló diferencias significativas entre estaciones en la densidad aparente del suelo y el contenido volumétrico de agua, tanto en condiciones de campo como en capacidad de campo ( $p$ $<0.001$ en los tres casos). Las pruebas de LSD y el test $U$ de Mann Whitney mostraron que el contenido volumétrico de agua en las dos condiciones, así como el porcentaje de materia orgánica edáfica fueron significativamente mayores en el bosque de E1 con respecto a las demás estaciones (Figura 3a). También en E1 la densidad aparente del suelo fue significativamente menor en comparación con las otras estaciones (Figura 3b).

Aunque dentro de cada estación se registraron cambios en el CMOE y en la densidad aparente del suelo con la profundidad (Figura 3c), así como en el contenido volumétrico de agua presente en condiciones de campo y en capacidad de campo, estos no fueron significativos de acuerdo con los análisis de varianza y la prueba de SRH $(p>0.05$ en todos los casos). Así mismo, estas pruebas estadísticas indicaron que las variables edáficas no presentaron diferencias significativas en las interacciones profundidad-estación ( $p>0.05$ en todos los casos).

El volumen deficitario de agua, relativizado a la capacidad de campo del suelo, mostró ser función logarítmica del CMOE con un nivel de ajuste significativo $\left(r^{2}=0.34, p<0.01\right)$; por lo tanto, el volumen deficitario de agua (medido como porcentaje del volumen de saturación), fue menor cuando el porcentaje de materia orgánica edáfica 

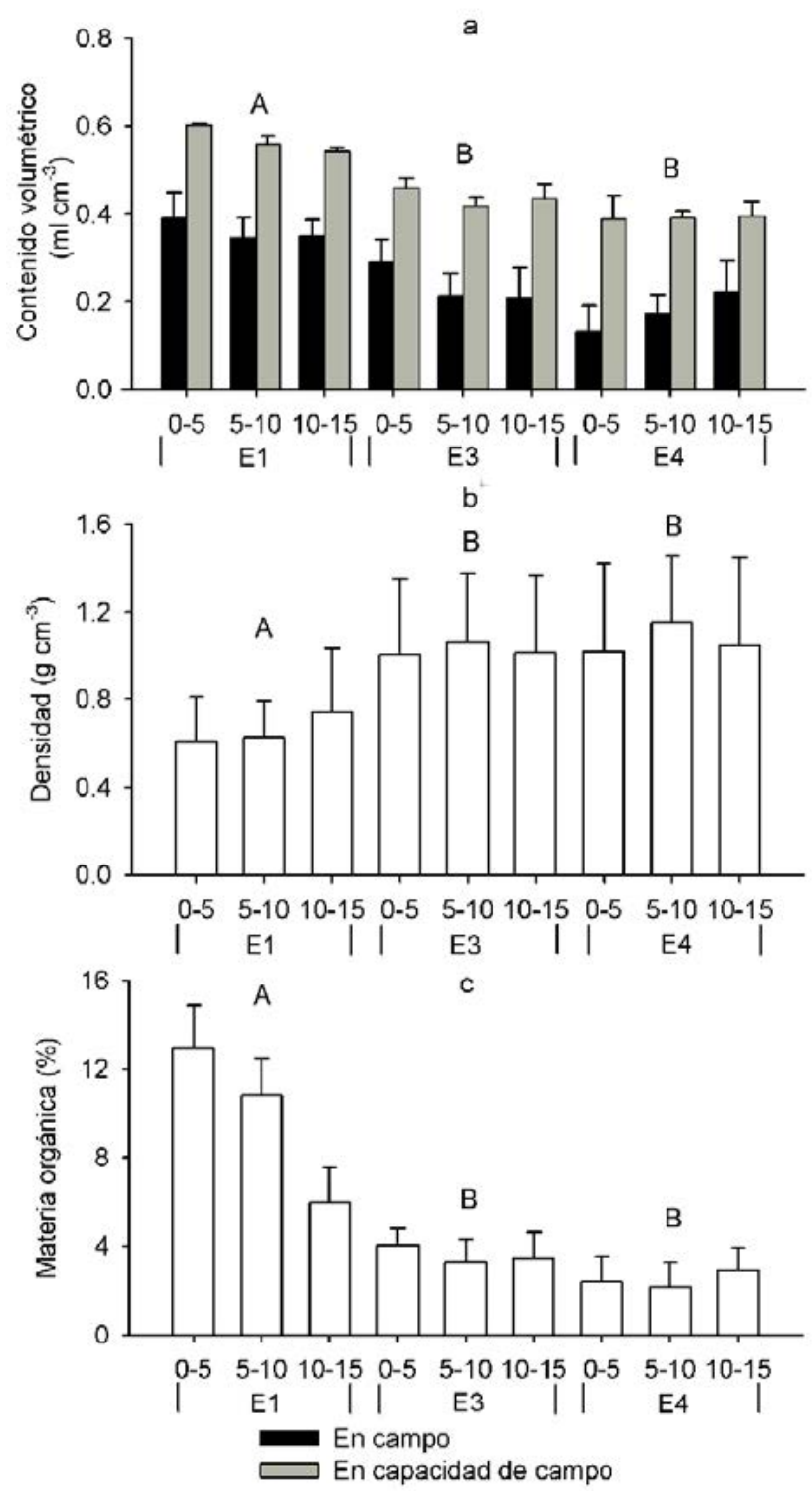

Figura 3. Contenido volumétrico de agua en condiciones de campo y en capacidad de campo (a), densidad aparente (b) y el porcentaje de materia orgánica edáfica (c) a profundidades de 0 a $5 \mathrm{~cm}$, 5 a $10 \mathrm{~cm}$ y 10 a $15 \mathrm{~cm}$. Cerca del nacimiento del caño (E1), en el lugar donde se construyó la planta de tratamiento de aguas residuales del municipio (E3) y en la reserva El Caduceo (E4). Las barras señalan el error estándar. Las letras mayúsculas indican diferencias estadísticas significativas entre estaciones $(\mathrm{p}<0.05)$. fue mayor (Figura 4a). No obstante, el volumen neto de agua requerido por los suelos de todas las estaciones para saturarse fue relativamente similar e independiente del porcentaje de materia orgánica (Figura 4b). La figura 4a muestra que los suelos boscosos y con alta cobertura de hojarasca de E1, comparados con los suelos de E3 y de E4, requieren para saturarse un porcentaje menor de agua en relación con el volumen de agua que pueden contener. Así mismo, el suelo de E3, que tiene una baja cobertura de hojarasca, requiere un
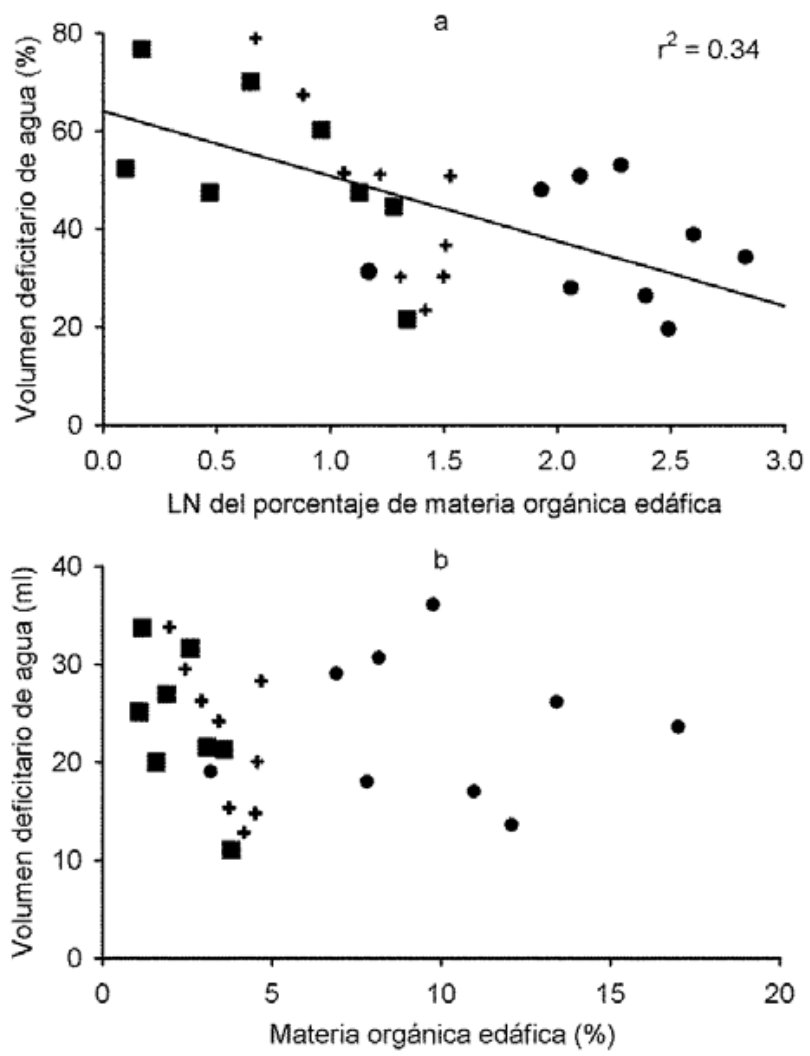

Figura 4. Relación entre el logaritmo natural (LN) del porcentaje de materia orgánica edáfica y el porcentaje de volumen deficitario de agua en condiciones de campo (a) y entre el porcentaje de materia orgánica edáfica y el volumen deficitario de agua en condiciones de campo (b). Los círculos corresponden a bosques riparios de E1; las cruces, a los bosques riparios de E3 y los cuadrados, a los pastizales riparios de E4 
porcentaje menor de agua para saturarse en relación con el suelo de pastizal de E4.

\section{Estructura trófica de los artrópodos del suelo superficial}

La estructura de la fauna de artrópodos asociados a los suelos boscosos la conformaron principalmente las familias Formicidae (hormigas), Forficulidae (tijeretas), Scarabaeidae (escarabajos del estiércol) y Curculionidae (gorgojos). Dichas familias representaron respectivamente el $71 \%, 5.2 \%, 4.6 \%$ y $3.8 \%$ de la biomasa total de artrópodos. La fauna de artrópodos asociados a pastizal la conformaron principalmente las familias Formicidae (87\%) y los saltamontes de la familia Gryllidae (4\%). Una especie de hormiga depredadora del género $\mathrm{Pa}$ chycondyla presentó una biomasa muy alta, tanto en suelos de bosque como de pastizal $(58.7 \%$ y $10.2 \%$, respectivamente), al igual que una especie de hormiga omnívora del género Solenopsis (1.5\% y $58.9 \%$, respectivamente). La alta biomasa de $\mathrm{Pa}$ chycondyla sp obedece a su relativo gran tamaño $(1.8 \mathrm{~mm})$ y abundancia $(1,9$ y 22 individuos en $\mathrm{E} 4$, E1 y E3, respectivamente), mientras que la de Solenopsis sp está dada por su alta abundancia (5, 26, 42 y 310 individuos en E2, E1, E3 y E4, respectivamente), ya que su tamaño es de $0.2 \mathrm{~mm}$.

Los saprófagos y los fungívoros exhibieron mayor representación en biomasa en suelos de bosque que en pastizal ( $13 \%$ vs. $3 \%$ y $9 \%$ vs. $2 \%$, respectivamente), mientras que los fitófagos lo hicieron mayormente en pastizales ( $1 \%$ vs. $9 \%$ ). Los depredadores, por su parte, fueron más dominantes en suelos de bosque que en pastizal (65.3\% vs. $23.1 \%$, respectivamente) y los omnívoros lo fueron en el pastizal (61.9\% vs. 9.7\%, respectivamente). Los consumidores primarios del pastizal, conformados por fitófagos, saprófagos y fungívoros, representaron el $74 \%$ de la biomasa y los consumidores secundarios, compuestos por depredadores y parasitoides, el 25\%. En los suelos de los bosques se presentó una estructura trófica invertida, pues los consumidores secundarios conformaron el $66 \%$ y los primarios el $23 \%$. Los omnívoros, que incluyen a consumidores primarios y secundarios, tuvieron mayor biomasa en pastizal que en bosque (62\% vs. 10\%) (Figura 5). Los saprófagos en el suelo del bosque estuvieron representados por las familias Forficulidae, Scarabaeidae, Phoridae (2\%), Sphaeroceridae $(0.3 \%)$, Termitidae $(0.3 \%)$, Nitidulidae $(0.2 \%)$ y Curculionidae $(0.6 \%)$. En el pastizal, las familias de saprófagos fueron Scarabaeidae $(0.8 \%)$, Curculionidae $(0.4 \%)$, Termitidae $(0.2 \%)$, Phoridae (0.2\%) y Ephydridae (0.2\%).

\section{DISCUSIÓN}

\section{Contenido de materia orgánica, densidad aparente y variables hídricas del suelo según condiciones del uso del suelo}

El CMOE encontrado en suelos de bosque y extrapolado a una profundidad de 0-20 cm (9.3 y 5.8 $\mathrm{kg} \mathrm{m}^{-2}$ para E1 y E3, respectivamente) se encuentra

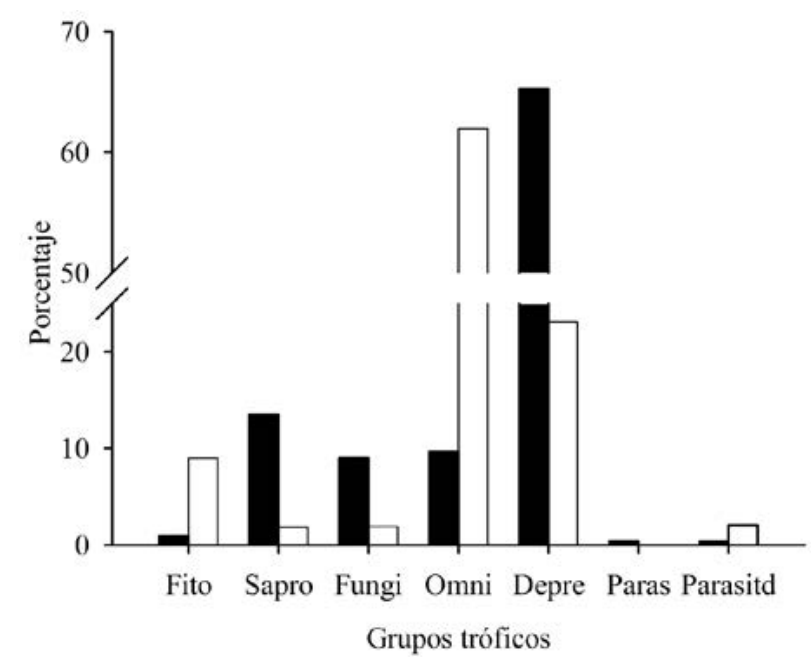

Figura 5. Organización trófica con base en el porcentaje de biomasa de los grupos de artrópodos presentes en los suelos de bosque (barras negras) y pastizales (barras blancas). Fito (fitófago), Sapro (saprófago), Fungi (fungívoro), Depre (depredador), Paras (parásito), Parasitd (parasitoide). 
cerca a la media de 35 muestras de suelo de bosques tropicales $\left(8.2 \mathrm{~kg} \cdot \mathrm{m}^{-2}\right.$, desviación estándar 4.6) (Jobbagy \& Jackson, 2000). El CMOE del pastizal $\left(4.5 \mathrm{~kg} . \mathrm{m}^{-2}\right)$ se encuentra cerca al promedio encontrado en 36 muestras de suelo de pastizales tropicales $\left(5.8 \mathrm{~kg} . \mathrm{m}^{-2}\right.$, desviación estándar 3.1) por Jobbagy \& Jackson (2000).

De manera similar a lo encontrado en el presente trabajo, se han reportado diferencias en la concentración de materia orgánica en los horizontes del suelo, atribuibles a la cobertura vegetal y al uso del suelo (Porto et al., 2003). No obstante, la ausencia de diferencias significativas en el CMOE entre profundidades y entre la interacción profundidad-estación sugiere que los tipos de cobertura vegetal evaluados no provocan cambios importantes en los primeros $15 \mathrm{~cm}$ de suelo.

Los CMOE y las densidades aparentes de los suelos en las estaciones de muestreo indican que a mayor CMOE se incrementa la infiltración y la conductividad hidráulica hacia los estratos inferiores. Estas relaciones ya han sido establecidas ampliamente en la literatura (Critchley \& Bruijnzeel, 1996; Cook et al., 1998; Gol, 2009). En dicho contexto es evidente que al presentar los bosques mayores $\mathrm{CMOE}$ (E1, E2, E3), tienen un rol más importante en la regulación hídrica que los pastizales (E4). Se destacan, no obstante, las diferencias significativas en CMOE que se presentaron entre los bosques y que sugieren que aquellos menos productivos (estación E3) generarían una dinámica de flujo subsuperficial y regulación hídrica mucho más limitada que los bosques con alto aporte de materia orgánica (estación E1).

El CMOE es una variable conservativa. En ausencia de erosión, dicha materia orgánica está compuesta en su mayoría por formas refractarias con tasas de recambio de décadas o siglos (Conant et al., 2011). Por eso, aunque se encontró cierta correspondencia entre el porcentaje de cobertura de hojarasca superficial del suelo y el CMOE, es importante considerar que sus concentraciones dependen de relaciones multianuales entre el manejo histórico del suelo, la productividad por encima y por debajo del suelo, los organismos detritívoros y descomponedores y las salidas por descomposición (Schmidt et al., 2011).

Por otra parte, la textura del suelo afecta la relación entre materia orgánica y retención de agua. Sin embargo, independiente de la textura, siempre habrá un incremento de la retención de agua cuando aumente el contenido de materia orgánica en suelos con porcentajes superiores al 5\% (Rawls et al., 2003). Según esto, el incremento de la continuidad de la oferta hídrica del caño Camoa no obedecería a una mayor cobertura boscosa riparia per se, sino al incremento de la edad del bosque secundario y al mantenimiento de áreas de este ecosistema poco intervenidas que aseguren la acumulación de materia orgánica en el suelo mediante el aumento de su productividad. De manera similar, tras una reforestación con árboles nativos el establecimiento de las propiedades hídricas del suelo estaría íntimamente ligado al avance del proceso sucesional (Wei et al., 2007).

En general, cuando existe un mayor CMOE se espera una menor probabilidad de escorrentía, pues la materia orgánica incrementa la infiltración del agua hacia estratos inferiores (Porto et al., 2003; Kirkby, 1998; Grazhdani \& Shumka, 2007). Así mismo, los bosques tienen tasas ligeramente más altas de evapotranspiración que los pastos (Cook et al., 1998) y coeficientes de intercepción mayores ( $27 \%$ vs. $22 \%$, respectivamente), si se incluye tanto el dosel como el estrato superficial (San José \& Montes, 1992; Seyffarth, 1995; Krushe et al., 2011; Tsiko et al., 2011). Según esto, los coeficientes de escorrentía son más altos en pastizales que en bosques (Treviño-Garza et al., 2002). Sin embargo, nuestros resultados y los de otros autores indican que el papel de la escorrentía superficial en los bosques estaría subestimado. Durante el periodo seco, los suelos de bosque presentan contenidos de humedad superiores a los pastizales (James et al., 2003). En bosques de pino se generan mayores niveles de escorrentía que en pastizales (Wei et al., 2007). Además, se han reportado, contra lo esperado, mayores potenciales de escorrentía en áreas con vegetación natural que en áreas cultivadas (Kirkby, 1998; Krishnaswamy et al., 2001; Wei et 
al., 2007). Kavian et al. (2014) encontraron mayores niveles de escorrentía en bosques que en cultivos de maíz y plantaciones de naranja como consecuencia de una mayor humedad antecedente del suelo en los bosques. Dichos resultados así como los de otros estudios (James et al., 2003; Liu et al., 2011) muestran que la humedad del suelo es un factor importante en condicionar las respuestas hidrológicas, pues reduce la infiltración y favorece la escorrentía.

Independientemente del CMOE y de la capacidad de campo, los suelos evaluados en el presente estudio mostraron potenciales similares para generar escorrentía. Sin embargo, también se encontró que los suelos con mayores contenidos de materia orgánica requirieron un menor volumen de agua para saturarse y generar escorrentía, a pesar de tener una capacidad de campo considerable. Aún no hay estudios que expliquen estos resultados, ni que definan los mecanismos que regulan los elevados niveles de escorrentía en suelos de bosques.

Kavian et al. (2014) sugieren que la hojarasca sobre la superficie del suelo disminuye la evaporación y la pérdida de humedad del suelo, lo que a su vez favorece la escorrentía en bosques en relación con otras coberturas, como se discutió anteriormente. Sin embargo, los bosques tienen altos coeficientes tanto de evapotranspiración como de intercepción (Cook et al., 1998; San José \& Montes, 1992; Seyffarth, 1995; Krushe et al., 2011; Tsiko et al., 2011). Los resultados del presente trabajo sugieren en cambio que el aumento de la materia orgánica edáfica asociada a las coberturas

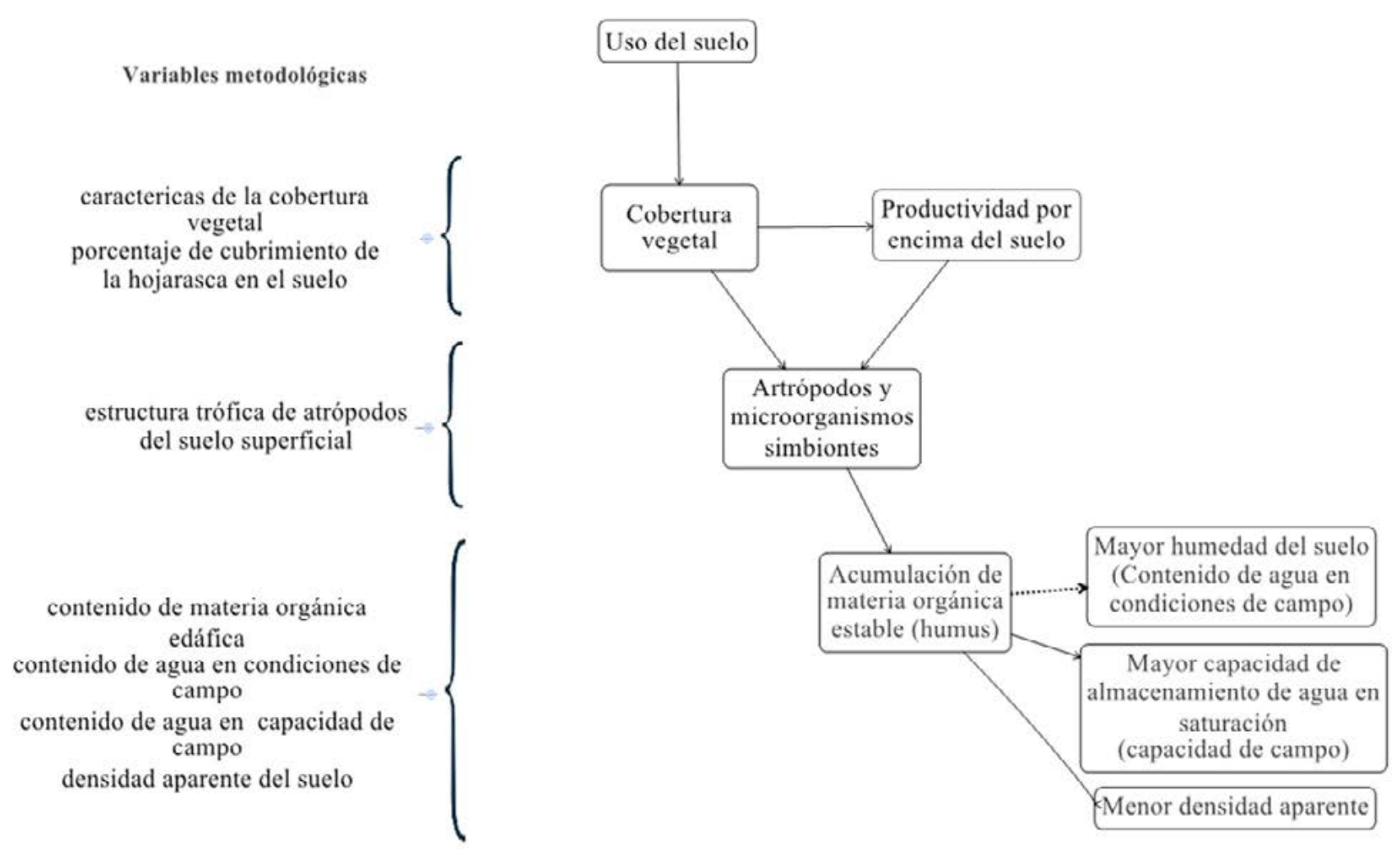

Figura 6. Modelo conceptual y metodológico de las relaciones entre el uso del suelo, los artrópodos y las propiedades físicas e hidrológicas del suelo consideradas en el estudio. Las líneas continuas indican relaciones entre las variables plenamente definidas y la línea discontinua relaciones hipotéticas. 
boscosas probablemente provoca un incremento combinado de la capacidad espacial de contener agua (Lewis et al., 2003) y de la capacidad de retención temporal, hipótesis que requerirá posteriores comprobaciones (Figura 6).

Por otra parte, el ajuste logarítmico encontrado sugiere que ambos tipos de retención de agua aumentan aceleradamente cuando el CMOE es del $0-6 \%$, y con menor rapidez cuando dicha materia orgánica es del 6-14\%. Esto implica que en el intervalo bajo de $\mathrm{CMOE}$, pequeños aportes de materia orgánica mejorarían ostensiblemente la retención espacial y temporal de agua. Similarmente, Rawls et al. (2003) encontraron alta sensibilidad en la retención de agua del suelo con el aumento de la materia orgánica cuando el contenido inicial de esta última fue inferior al $5 \%$ en todas las texturas de suelo. Sin embargo, a diferencia de los suelos arenosos o limosos, los suelos arcillosos con contenidos de materia orgánica iniciales inferiores al 5\%, disminuyeron la capacidad de retención de agua con incrementos de la materia orgánica (Rawls et al., 2003). Por lo anterior, en el manejo de las cuencas con suelos arcillosos se deben evaluar las condiciones iniciales de materia orgánica.

En suma, los resultados sugieren que los bosques con alta productividad presentan mayores capacidades de campo y de retención de agua, así como potenciales de escorrentía relativamente altos, independientemente de la textura de sus suelos. Dichas características ocurrirán si la productividad de los bosques se asocia con valores altos de CMOE (7-17\%).

Hay que destacar que una escorrentía relativamente alta en los bosques no implica necesariamente niveles mayores de erosión (Wei et al., 2007), pues la intercepción de la precipitación por el follaje y la hojarasca juegan un papel importante en la disminución de la fuerza cinética asociada a la caída de las gotas (Zhou et al., 2006). Al principio del periodo lluvioso, los suelos de los pastizales se saturan más rápidamente que los de los bosques (James et al., 2003), probablemente como consecuencia de menores flujos subsuperficiales y una menor intercepción. Por ello, los bosques ejercen un papel regulatorio de la escorrentía y la inundación durante los periodos de precipitación intensa (Krishnaswamy et al., 2001). Sin embargo, dicho poder regulatorio decrecería con un mayor nivel de saturación del suelo boscoso.

Los suelos de bosques productivos aportan agua al cauce mediante la vía continua y lenta del flujo subsuperficial y mediante la vía esporádica de la escorrentía superficial. Los suelos de pastizales y de bosques poco desarrollados, por su parte, aportarían agua principalmente mediante escorrentía superficial, pues el flujo subsuperficial estaría limitado por las mayores densidades aparentes del suelo (ONU, 2006).

Aunque en el presente estudio se analizaron suelos riparios boscosos y con pastizales, en las riberas del caño Camoa y en la mayoría de ríos de la Orinoquia se encuentran diferentes tipos de cultivos, entre los que se destacan los de palma africana, arroz y patilla. Las coberturas agropecuarias promueven particularmente altos niveles de escorrentía que implican pérdida de materia orgánica edáfica por la erosión asociada (Wei et al., 2007; Kavian et al., 2014). En Costa Rica, por ejemplo, se ha detectado que las cuencas que experimentaron altas tasas de conversión de bosques por áreas agropecuarias presentaron mayor frecuencia e intensidad en eventos de escorrentía de alta magnitud, así como mayores niveles de descarga de sedimentos (Krishnaswamy et al., 2001). Todo lo anterior muestra que el manejo de las cuencas y de las áreas riparias debe tender al mantenimiento de áreas boscosas maduras y productivas, así como a la disminución de tensores como la contaminación, la tala y el pastoreo.

\section{Estructura trófica de los artrópodos del suelo superficial y su rol en la vía detrítica}

La alta representación de los depredadores en los bosques estudiados queda relegada a un segundo 
plano, si no se considera la fuerte dominancia en número y biomasa de las hormigas del género Pachycondyla. Estas hormigas tendrían la capacidad de movilizarse y depredar a ras de suelo y en los estratos herbáceo, arbustivo y arbóreo debido a su gran tamaño, lo que las ubicaría como consumidoras secundarias en varios estratos de la vegetación. Sin considerar esta especie depredadora, saprófagos, omnívoros y fungívoros pasan a ser los grupos dominantes en biomasa (33\%, 23\%, 22\%, respectivamente), seguidos luego por los depredadores, fitófagos y parasitoides $(16 \%, 2 \%$ y $1 \%$, respectivamente). Así mismo, la vía trófica detrítica adquiere mayor relevancia dada la alta biomasa de saprófagos y fungívoros. Esto se refleja en la apreciable representación de las familias de hábito saprófago como Forficulidae, Scarabaeidae, Phoridae, Termitidae y Sphaeroceridae (13\%, 11\%, 5\%, $1 \%$ y $1 \%$ de la biomasa, respectivamente).

En cuanto al pastizal estudiado, la baja representación encontrada del componente saprofítico podría explicarse por la menor disponibilidad de hojarasca y troncos muertos. Además, la principal vía saprofítica en los pastizales no estaría asociada al follaje y a la capa más superficial del suelo, sino a la rizósfera, donde intervienen nemátodos, lombrices, macro y microartrópodos (Stanton, 1988). Lo anterior sugiere que en pastizales con árboles dispersos la hojarasca y los sustratos leñosos tardarían más tiempo en ser ubicados y degradados por los organismos saprófagos en comparación con bosques, donde hay mayor abundancia tanto de sustratos leñosos como de saprófagos. De hecho, la complejidad estructural influencia considerablemente la abundancia de invertebrados y la estructura de la comunidad (Olson et al., 1995).

Por su parte, las actividades de ganadería incidirían en la organización trófica de los artrópodos del pastizal estudiado. La alta dominancia de hormigas del género Solenopsis es típica de ecosistemas húmedos disturbados de tierras bajas (Risch \& Carroll, 1983) y se ve reflejada en la mayor biomasa encontrada de los omnívoros con respecto a los fitófagos.
Los géneros y familias de artrópodos encontrados facilitan la degradación de la materia orgánica al consumirla directamente o con la ayuda de simbiontes o al facilitar el establecimiento de hongos y bacterias en el suelo. En los bosques, las termitas degradan la celulosa y la lignina con la ayuda de bacterias y protistas simbiontes (Donovan et al., 2004). Así mismo, los gorgojos de las cortezas (Curculionidae, subfamilia Scolytinae; 8\% de la biomasa total) convierten la madera en fibras gruesas o polvo al cavar en los tejidos vegetales y facilitar la colonización de hongos simbiontes de los que se alimentan (Nair, 2007).

Los individuos de las familias Forficulidae (tijeretas), Phoridae y Sphaeroceridae intervienen sobre la materia orgánica semidescompuesta o descompuesta facilitando su degradación, mientras que la familia Scarabaeidae (escarabajos del estiércol) agiliza la degradación de heces animales. Las dietas omnívoras de las cucarachas BlatteIlidae y de las hormigas de los géneros Ectatomma, Solenopsis y Dolichoderus hacen suponer que estas actúan también sobre material muerto, vegetal o animal. En el Anexo 1 se listan los hábitos tróficos de los taxones de artropofauna capturados en la región del caño Camoa.

Los colémbolos (orden Collembola, 9.5\% de la biomasa) y los escarabajos fungívoros de las familias Corylophidae y Ptiliidae $(0.6 \%$ y $0.1 \%$ de biomasa, respectivamente) consumen activamente los hongos saprofíticos, aprovechando la dominancia de estos en la capa más superficial del suelo (Killham, 1994; Jonas et al., 2007). Otros grupos como las hormigas de los géneros Cyphomyrmex ( $0.1 \%$ de biomasa) y los gorgojos de las cortezas cultivan hongos (Currie et al., 2006; De Fine Licht \& Boomsma, 2010), y por lo tanto facilitan su establecimiento en el suelo. El género de hormigas Cheliomyrmex (4\%, de biomasa), por su parte, se alimenta de invertebrados subterráneos y, probablemente, del tegumento que remueve de algunos vertebrados usando sus mandíbulas en forma de garra (De Araújo et al., 2008). 
Los niveles de consumo intermedios de hongos por parte de los colémbolos pueden incrementar la cantidad de nitrógeno y fósforo disponible para las plantas mediante deposiciones fecales o estimulando la actividad de descomposición de hongos saprofíticos (Gange, 2000; Jonas et al., 2007). La acción de termitas, escarabajos de las cortezas y de otros grupos detritívoros promueve la degradación de moléculas complejas y la liberación de nitratos, calcio y carbono orgánico a tasas significativamente más altas que los microorganismos (Pramanik et al., 2001; Nair, 2007).

La acción de artrópodos, hongos y bacterias también media en los procesos de formación de materia orgánica estable o humus, que es quizá la fracción de la materia orgánica más importante en incrementar la capacidad de retención de agua, dado su papel estabilizador de agregados del suelo (Lewis et al., 2003). Así mismo, los artrópodos de mayor tamaño, que son consumidos por anfibios, reptiles, aves y mamíferos insectívoros, median en la reincorporación de la materia orgánica a las cadenas tróficas (Naranjo \& Chacón de Ulloa, 1997; Sánchez-Núñez \& Amat-García, 2005).

Aunque las comunidades de artrópodos presentan cambios estacionales apreciables en la composición y diversidad de especies (Erwin \& Scott, 1980; Recher et al., 1996), las fluctuaciones estacionales en la estructura trófica son mucho más limitadas (Mackay \& Kalf, 1968, Schowalter \& Ganio, 1998). De la misma manera, las comunidades de artrópodos asociadas a pastizales o riachuelos han mostrado no presentar cambios estacionales significativos en su organización trófica (Evans \& Murdoch, 1968; Motta \& Uieda, 2004). No obstante, la estructura trófica identificada en los bosques presentaría una menor variabilidad entre estaciones con respecto a los pastizales, teniendo en cuenta su mayor dependencia de la vía detrítica (Motta \& Uieda, 2004) y la disposición constante en los suelos boscosos de sustratos en descomposición como hojarasca, troncos y cadáveres, independientemente de la estacionalidad.

\section{CONCLUSIONES}

La relación significativa encontrada entre el porcentaje de materia orgánica edáfica y el volumen deficitario de agua, aun al final del periodo lluvioso, indica que el efecto de esta variable conservativa tiene una impronta fuerte sobre el contenido de agua. Por otro lado, independientemente de la textura del suelo, la capacidad de retención de agua siempre se incrementa cuando el contenido de materia orgánica de partida es mayor al 5\% (Rawls et al., 2003). Como consecuencia, el manejo de las áreas riparias debe propender por mantener bosques desarrollados de alta productividad y no bosques per se. Esto permitiría regular el exceso hídrico durante los periodos lluviosos y mantener un mayor volumen y continuidad durante los periodos secos. Esta hipótesis, sin embargo, debe verificarse en diferentes periodos climáticos.

La regulación de flujos hídricos es un SE clave para el país, que no obstante es vulnerable por el grado de deforestación de las cuencas y la variabilidad climática en un contexto de incertidumbre debido a cambios globales. Por otra parte, las valoraciones económicas de este SE, los modelos hidrológicos y la ordenación de cuencas tienen poco en cuenta los notables cambios de regulación hídrica que pueden asociarse al grado de desarrollo de los bosques (Loaiza \& Valentijn, 2011; Reyes, 2014). Es necesario por lo tanto que los ejercicios de ordenación y valoración de las cuencas hidrográficas consideren el grado de desarrollo de los bosques riparios y sus CMOE asociados.

La contrastante representación de los saprófagos en los bosques y en el pastizal estudiado evidencia una mayor disposición de sustratos como hojarasca, troncos y cadáveres de animales en los bosques. También sugiere la importancia de estos organismos para la degradación de moléculas simples y complejas y para el mejoramiento de la capacidad de retención e infiltración del agua en el suelo, puesto que dichas moléculas pueden ser transformadas y secuestradas temporalmente en forma de humus. De la descomposición de la 
materia orgánica depende la mineralización y el reciclaje de los nutrientes. Ambos procesos, degradación y descomposición, serían favorecidos por una organización trófica bien estructurada de artrópodos, y muy seguramente de microorganismos.

Los resultados obtenidos muestran tendencias claras, sin embargo, el presente estudio no considera la variabilidad climática e hidrológica, por lo que es recomendable corroborar estos resultados en futuros estudios con mayor amplitud espacial y temporal.

\section{AGRADECIMIENTOS}

Agradecemos a la Dirección Curricular de Posgrado del Departamento de Biología y a la Facultad de Ciencias de la Universidad Nacional de Colombia por apoyar económicamente la salida de campo de la asignatura de posgrado Ecología de Ecosistemas durante la cual se realizó la presente investigación. Agradecemos especialmente a Juanita Mora por su apoyo y colaboración prestados en la fase de campo, a Miguel Suárez por facilitar la realización del estudio en la reserva El Caduceo, a German Amat-García y Fernando Fernández, del Instituto de Ciencias Naturales, por su colaboración en la determinación taxonómica de los insectos y a William Pinto, del Ministerio de Medio Ambiente y Desarrollo Sostenible, por la elaboración del mapa del área de estudio.

\section{REFERENCIAS BIBLIOGRÁFICAS}

Alcaldía de San Martín de los Llanos. (1996). Plan de manejo, protección, conservación y reforestación de la microcuenca del caño Camoa en el municipio de San Martín. San Martín, Meta: Alcaldía de San Martín de los Llanos. 53 p.

Bashour, I., \& Sayegh, A. (2007). Methods of analysis for soils of arid and semi-arid regions. Rome: Food and Agriculture Organization of the United Nations. $119 \mathrm{p}$.
Blinn, R., \& Kilgore, A. (2001). Riparian Management Practices. Journal of Forestry, 8, 11-17.

Castaño-Meneses, G., Palacios-Vargas, J., \& Cutz-Pool, L. (2004). Feeding habits of Collembola and their ecological niche. Anales del Instituto de Biología Serie Zoología, 75, 135-142.

Cloarec, A., \& Rivault, C. (1991). Age related changes in foraging in the German cockroach (Dictyoptera: Blattellidae). Journal of Insect Behavior, 4, 661-673.

Conant, R., Ryan, M., Agren, G., Birge, H., Davidson, E., Eliasson, P., Evans, S., Frey, S., Giardina, C., Hopkins, F., Hyvönen, R., Kirschbaum, M., LavaIlee, J., Leifeld, J., Parton, W., Megan-Steinweg, J., Wallenstein, M., Martin-Wetterstedt, J. Å. \& Bradford, M. (2011). Temperature and soil organic matter decomposition rates - synthesis of current knowledge and a way forward. Global Change Biology, 17, 3392-3404.

Cook, P., Hatton, T., Pidsley, D., Herczeg, A., Held, A., O'Grady, A., \& Eamus, D. (1998). Water balance of a tropical woodland ecosystem, Northern Australia: a combination of micro, meteorological, soil physical and groundwater chemical approaches. Journal of Hydrology, 210, 161-177.

Corporación para el Desarrollo Sostenible del Área de Manejo Especial La Macarena (Cormacarena). (2004). Plan de acción trienal 2004 - 2006. Villavicencio: Cormacarena. $114 \mathrm{p}$.

Critchley, W., \& Bruijnzeel, L. (1996). Environmental impact of converting moist tropical forest to agriculture and plantations. IHP Humid Tropics Programme Series No 10. Holanda: UNESCO. 48 p.

Currie, C., Poulsen, M., Mendenhall, J., Boomasma, J., \& Billen, J. (2006). Coevolved crypts and exocrine glands support mutualistic bacteria in fungus-growing ants. Science, 311, 81-83.

De Araújo, F., Ferreira, C., \& Viegas, K. (2008). Subterranean ants (Hymenoptera, Formicidae) as prey of fossorial reptiles (Reptilia, Squamata: Amphisbaenidae) in Central Brazil. Papéis Avulsos de Zoologia, 48, 329-334.

De Fine Licht, H., \& Boomsma, J. (2010). Forage collection, substrate preparation, and diet composition in 
fungus-growing ants. Ecological Entomology, 35, 259-269.

Dominati, E., Patterson, M., \& Mackay, A. (2010). A framework for classifying and quantifying the natural capital and ecosystem services of soils. Ecological Economics, 69, 1858-1868.

Donovan, S., Purdy, K., Kane, M., \& Eggleton, P. (2004). Comparison of Euryarchatea strains in the guts and food-soil of the soil-feeding termite Cubitermes fungifaber across different soil types. Applied and Environmental Microbiology, 70, 3884-3892.

Elliot, E., Heil, J., Kelly, E., \& Manger, H. (1999). Soil structural and others physical properties. En G. Robertson, D. Coleman, C. Bledsoe, P. Sollins (eds.). Standard soil methods for long term ecological research (pp. 55-73). Nueva York: Oxford University Press.

Erwin, T., Scott, J. (1980). Seasonal and size patterns, trophic structure and richness of coleopteran in the tropical arboreal ecosystem: the fauna of the tree Luehea seemanni Triana and Planch in the canal of Panama. The Coleopterists' Bulletin, 34, 305-322.

Evans, F., \& Murdoch, W. (1968). Taxonomic composition, trophic structure and seasonal occurrence in a grassland insect community. Journal of Animal Ecology, 37, 259-273.

Fernández, F. (ed.) (2003). Introducción a las hormigas de la región neotropical. Bogotá: Instituto Humboldt. 398 p.

Gange, A. (2000). Arbuscular mycorrhizal fungi, CoIlembola and plant growth. Trends in Ecology \& Evolution, 15, 369-372.

Gol, C. (2009). The effects of land use change on soil properties and organic carbon at Dagdami river catchment in Turkey. Journal of Environmental Biology, 30, 825-830.

Grazhdani, S., \& Shumka, S. (2007). An approach to mapping soil erosion by water with application to Albania. Desalinization, 213, 263-272.

Guerrero, P., \& Arias, M. (1984). La política agraria en el municipio de San Martín (Tesis de pregrado, Historia). Bogotá: Departamento de Historia, Facultad de Ciencias Humanas, Universidad Nacional de Colombia. $124 \mathrm{p}$.
Huang, J., Zhan, J., Yan, H., Wu, F., \& Deng, X. (2013). Evaluation of the impacts of land use on water quality: a case study in the Chaohu Lake basin, The Scientific World Journal, 2013, 7 p.

Instituto de Investigación de Recursos Biológicos Alexander von Humboldt (IAvH). (2006). Proyecto: Biodiversidad y desarrollo en ecorregiones estratégicas de Colombia, Orinoquía. Recuperado de http://www.humboldt.org.co/proyecto_orinoquia/

Instituto Geográfico Agustín Codazzi (IGAC). (1996). Diccionario Geográfico de Colombia ( $3^{\text {a }}$ edición). Santafé de Bogotá: Horizonte Impresos. 2521 p.

James, S., Partel, M., Wilson, S., \& Peltzer, D. (2003). Temporal heterogeneity of soil moisture in grassland and forest. Journal of Ecology, 91, 234-239.

Jobbagy, E., \& Jackson, R. (2000). The vertical distribution of soil organic carbon and its relation to climate and vegetation. Ecological Applications, 10, 423-436.

Jonas, J., Wilson, G., White, P., \& Joern, A. (2007). Consumption of mycorrhizal and saprophytic fungi by Collembola in grassland soils. Soil Biology \& Biochemistry, 39, 2594-2602.

Kavian, A., Azmoodeh, A., Solaimani, K. (2014). Deforestation effects on soil properties, runoff and erosion in northern Iran. Arabian Journal of Geosciences, 7, 1941-1950.

Killham, K. (1994). Soil ecology. Great Britain: Cambridge University Press. 242 p.

Kirkby, M. (1998). Evaluation of pilot runoff and erosion forecast using the CSEP and medrush models. En J. Boardman, \& D. F. Favis-Mortlock (eds.). Modelling soil erosion by water (pp. 33-42). Berlin: Springer-Verlag.

Kluber, M., Olson, D., Puettmann, K. (2008). Amphibian distribution in riparian and upslope areas and their habitat associations on managed forest landscapes in the Oregon Coast Range. Forest Ecology and Management, 256, 529-535.

Krishnaswamy, J., Halpin, P., \& Richter, D. (2001). Dynamics of sediment discharge in relation to landuse and hydro-climatology in a humid tropical watershed in Costa Rica. Journal of Hydrology, 253, 91-109. 
Krushe, A., Ballester, M., \& Leite, N. (2011). Hydrology and biochemistry of terra firme lowland tropical forest. En D. Levia, D. Moses, \& T. Tanaka (eds.). Forest hydrology and biogeochemistry. Synthesis of past research and future direction (pp. 187-202). London: Springer Dordrecht Heidelberg.

Laurence, B. (1955). The ecology of some British Sphaeroceridae (Borboridae, Diptera). Journal of Animal Ecology, 24, 187-199.

Lavelle, P. (1997). Faunal activities and soil processes: Adaptive strategies that determine ecosystem function. Advances in Ecological Research, 27, 93-132.

Le Maitre, D., Kotzee, D., \& O'Fareel, P. (2014). Impacts on land-cover change on the water flow regulation ecosystem service: Invasive alien plants, fire and their policy implications. Land Use Policy, 36, 171-181.

Lewis, L., Clark, L., Krapf, R., Manning, M., Staats, J., Subirge, T., Townsend, L., \& Ypsilantis, B. (2003). Riparian area management: Riparian wetland soiIs. Denver, Colorado: Bureau of Land Management. $109 \mathrm{p}$.

Liu, H., Lei, T., Zhao, J., Yuan, C., Fan, Y., Qua, L. (2011). Effects of rainfall intensity and antecedent soil water content on soil infiltrability under rainfall conditions using the run off-on-out method. Journal of Hydrology, 396, 24-32.

Loaiza, J., \& Valentijn, R. (2011). Desarrollo de modelos hidrológicos y modelación de procesos superficiales. Gestión y Ambiente, 14, 23-32.

Mackay, R., Kalf, J. 1968. (1968). Seasonal variation in standing crop and species diversity of insect communities in a small Quebec stream. Ecology, 50, 101-109.

Martínez-Mena, M., López, J., Almagro, M., Boix-Fayos, V., \& Albaladejo, J. (2008). Effect of water erosion and cultivation on the soil carbon stock in a semiarid area of south-east Spain. Soil and Tillage Research, 99, 119-129.

Moran, M, D. (2003). Arguments for rejecting the sequential Bonferroni in ecological studies. Oikos, 100, 403-405.
Motta, R., \& Uieda, V. (2004). Diet and trophic groups of an aquatic insect community in a tropical stream. Brazilian Journal of Biology, 64, 809-817.

Nair, K. (2007). Tropical forest insect pests: Ecology, impact, and management. Cambridge: Cambridge University Press. 424 p.

Naranjo, L., \& Chacón de Ulloa, P. (1997). Diversidad de insectos y aves insectívoras de sotobosque en hábitats perturbados de selva Iluviosa tropical. Caldasia, 19, 507-520.

Olson, E., Engstromes, E., Doeringsfeld, M., \& Bellig, R. (1995). Abundance and distribution of macroinvertebrates in relation to macrophyte communities in a prairie marsh, Swan Lake, Minnesota. Journal of Freshwater Ecology, 10, 325-335.

Organización de las Naciones Unidas para la Educación la Ciencia y la Cultura-Programa Hidrológico Internacional para América Latina y el Caribe. (2006). Documento Técnico No 2. Balance hídrico integrado y dinámico en el Salvador-Componente evaluación de recursos hídricos. Montevideo: UNESCO PHI-LAC. 139 p.

Porto, J., López-Acevedo, M., \& Roquero, C. (2003). Edafología para la agricultura y el medio ambiente $\left(3^{a}\right.$ edición). Madrid: Ediciones Mundi Prensa. 929 p.

Pramanik, R., Sarkar, K., \& Joy, V. (2001). Efficiency of detritivore soil arthropods in mobilizing nutrients from leaf litter. Tropical Ecology, 42, 51-58.

Rawls, W., Pachepsky, Y., Ritchie, J., Sobecki, T., \& Bloodworth, H. (2003) Effect of soil carbon on soil water retention. Geoderma, 116, 61-76.

Recher, H., Majer, J., \& Ganesh, J. (1996). Seasonality of canopy invertebrate communitiess in eucalipt forest of Eastern and western Australia. Australian Journal of Ecology, 21, 64-80.

Reyes, M. (2014). Importancia económica de la provisión y regulación hídrica de los parques Nacionales de Colombia para los sectores productivos del país. Simposio 11. Reconocimiento de los servicios ecosistémicos de las áreas protegidas. II Congreso Colombiano de Áreas Protegidas. Bogotá: Parques Nacionales Naturales de Colombia. 
Risch, S., \& Carroll, C. (1983). Effect of a keystone predaceous ant, Solenopsis geminata on arthropods in a tropical agroecosystem. Ecology, 63, 1979-1083.

Rodríguez, J. (2006). Dinámica de la materia orgánica en la cabecera de una quebrada de montaña (Tesis de maestría, Biología). Bogotá: Departamento de Biología, Facultad de Ciencias, Universidad Nacional de Colombia. 96 p.

Rodríguez, J., Rojas-Suárez, F., \& Giraldo, D. (2010). Libro rojo de los ecosistemas terrestres de Venezuela. Caracas, Venezuela: Provita, Shell Venezuela, Lenovo. 324 p.

Rogers, L., Hinds, W., \& Bushbom, R. (1976). A general weight vs. length relationship for insects. Annals of the Entomological Society of America, 69, 387-389.

Sánchez-Núñez, D., \& Amat-García, G. (2005). Diversidad de la fauna de artrópodos terrestres del humedal Jaboque, Bogotá-Colombia. Caldasia, 27, 311-329.

San José, J., \& Montes, R. (1992). Rainfall partitioning by a semidecidous forest grove in the savannas of the Orinoco Llanos, Venezuela. Journal of Hydrology, 132, 249-262.

Scanlon, B., Reedy, R., Stonestrom, D., Prodic, D., \& Dennehys, K. (2005). Impact of land use and land cover change on groundwater recharge and quality in the southestern US. Global Change Biology, 11, 1577-1593.

Schmidt, M., Torn, M., Abiven, S., Dittmar, T., Guggenberger, G., Janssens, I, Klebber, M., Kögel-knabner, I., Lehmann, J., Manning, D., Nannipieri, P, Rasse, D., Weiner, S., \& Trumbore, S. (2011). Persistence of soil organic matter as an ecosystem property. $\mathrm{Na}$ ture, 478, 49-56.

Schowalter, T., \& Ganio, L. (1998). Vertical and seasonal variation in canopy arthropod communities in an old-growth conifer forest in southwestern Washington, USA. Bulletin of Entomological Research, 88, 633-640.

Schulte, E., \& Hopkins, B. (1996). Estimation of soil organic matter by weight Organic Matter (LOI) loss-on-ignition. En F. R. Magdoff, M. A. Tabatabai, \& E. A Hanlon (eds.). Soil Organic Matter: Analysis and Interpretation (pp. 21-31). Madison: Soil Science Society of America.

Seyffarth, J. (1995). Interceptação de chuva em cerrado sensu stricto sob diferentes regimes de queima (Tese de maestria, Mestre Ecologia). Brasilia: Universidad de Brasilia. 93 p.

Sokal, R., \& Rohlf, F. (1995). Biometry. The Principles and Practice of Statistics in Biological Research, 3rd Edition. W.H. New York: Freeman and Co. 887 p.

Stanton, N. (1988). The underground in grasslands. Annual Review of Ecology and Systematics, 19, 573-589.

Statpoint Technologies Inc. (1998). STATGRAPHICS 4.0 PLUS.

Treviño-Garza, E., Muñoz, C., Cavazos, C., \& Barajas-Chaves, L. (2002). Evaluación del flujo hídrico superficial en la Sierra de San Carlos. Tamaulipas. Ciencia UANL, 5. Disponible en http://eprints.uanl. $\mathrm{mx} / 500 /$

Triplehorn, C., \& Johnson, N. (2005). Borror and DeLong's Introduction to the Study of Insects ( $7^{\text {th }}$ Edition). Belmont, USA: Thomas Brooks/Cole. $863 \mathrm{p}$.

Tsiko, C., Makurira, H., Gerrits, A., \& Saavenije, H. (2011). Measuring forest floor and canopy interception in a Savannah ecosystem. Physics and Chemistry of the Earth, 47-48, 122-127.

Vörösmarty, C., Lettenmaier, D., Leveque, C., Meybeck, M., Pahl-Wostl, C., Alcamo, J., Cosgrove, H., Grassl, H., Hoff, H., \& Kabat P. (2004). Humans transforming the global water system. Eos, Transactions American Geophysical Union, 85, 509-520.

Wei, W., Chen, L., Fu, B., Huang, Z., Wu, D., \& Gui, L. (2007). The effect of land uses and rainfall regimes on runoff and soil erosion in the semi-arid loess hilly area, China. Journal of Hydrology, 335, 247-258.

Zhou, Z., Shangguan, Z., \& Zhao, D. (2006). Modeling vegetation coverage and soil erosion in the loess Plateau Area of China. Ecological Modelling, 198, 263-268. 


\section{Anexo 1.}

Categorías tróficas de los artrópodos encontrados en suelos de bosque y pastizal en la cuenca alta y media del caño Camoa. Categorías basadas en Laurence (1955), Cloarec y Rivault (1991), Castaño-Meneses et al. (2004), Donovan et al. (2004), Fernández (2003) y Triplehorn y Jhonson (2005).

\begin{tabular}{|c|c|c|c|}
\hline Orden & Familia & Género & Grupo trófico \\
\hline Acari & Todas & & Parasito \\
\hline Araneae & Todas & & Depredador \\
\hline Opiliones & & & Depredador o saprófagos \\
\hline Scorpiones & Todas & & Depredador \\
\hline Blattodea & Blattellidae & & Omnívoro \\
\hline Colembolla & Entomobridae & & Fungívoro \\
\hline Colembolla & Isotomidae & & Fungívoro \\
\hline Colembolla & Phoduridae & & Fungívoro \\
\hline Colembolla & Sminthuridae & & Fungívoro \\
\hline Coleoptera & Corylophidae & & Fungívoro \\
\hline $\begin{array}{l}\text { Coleoptera } \\
\text { (Subfamilia Scolytinae) }\end{array}$ & Curculionidae & & Fungívoro \\
\hline Coleoptera & Curculionidae & & Saprófago \\
\hline Coleoptera & Elateridae & & Fitófago \\
\hline Coleoptera & Nitidulidae & & Saprófago \\
\hline Coleoptera & Ptiliidae & & Fungívoro \\
\hline Coleoptera & Scarabeidae & & Saprófago \\
\hline Coleoptera & Staphilinidae & & Depredador \\
\hline Dermaptera & Forficulidae & & Saprófago \\
\hline Diptera & Cecidomyiidae & & Fitófago \\
\hline Diptera & Chironomidae & & \\
\hline Diptera & Drosophilidae & & Saprófago \\
\hline Diptera & Ephydridae & & Saprófago \\
\hline Diptera & Phoridae & & Saprófago \\
\hline Diptera & Sciaridae & & \\
\hline Diptera & Sphaeroceridae & & Saprófago \\
\hline Hymenoptera (Superfamilia Chalcidoidea) & Todas & & Parasitoide \\
\hline Hymenoptera & Braconidae & & Parasitoide \\
\hline Hymenoptera & Formicidae & Dolichoderus & Omnívoro \\
\hline Hymenoptera & Formicidae & Cheliomyrmex & Depredador \\
\hline Hymenoptera & Formicidae & Solenopsis & Omnívoro \\
\hline Hymenoptera & Formicidae & Hypoponera & Depredador \\
\hline Hymenoptera & Formicidae & Ectatomma & Omnívoro \\
\hline Hymenoptera & Formicidae & Pheidole & Fitófago \\
\hline Hymenoptera & Formicidae & Pachychondyla & Depredador \\
\hline Hymenoptera & Formicidae & Camponotus & Omnívoro \\
\hline Hymenoptera & Formicidae & Odontomachus & Depredador \\
\hline Hymenoptera & Formicidae & Cyphomyrmex & Fungívoro \\
\hline Hymenoptera & Formicidae & Acanthognathus & Depredador \\
\hline Isoptera & Termitidae & & Saprófago \\
\hline Neuroptera & Hemerobiidae & & Depredador \\
\hline Orthoptera & Grillidae & & Fitófago \\
\hline Thysanoptera & Phlaeothripidae & & Fungívoros o saprófagos \\
\hline
\end{tabular}

